\title{
Methodisches Entwickeln von Anforderungen in der Produktentwicklung
}

Young-Woo Song ${ }^{1}$, Frederike Kossack ${ }^{1}$, Beate Bender ${ }^{1}$

${ }^{1}$ Chair for Product Development,

Ruhr-Universität Bochum (RUB), Germany

\section{Abstract}

Product development projects are bound by objectives of various stakeholders. In early planning and design phases, these objectives can be very vague due to uncertainties with regard to knowledge and information about potential solutions as well as the design process. Moreover, time and cost requirments interact with product related requirements. Still, from the beginning of any design activity objectives must be translated into requirements to serve as a common information basis for the design process and to allow for the verification of the design solution. This paper presents a case study to exemplify typical problems in the development process caused by ill-defined requirements. In addition, approaches for a methodical procedure to address these problems are presented.

Keywords: Requirements Engineering, Product Development, Design Goals

\section{$1 \quad$ Einleitung}

Die Erweiterung des Produktbegriffs zum Produkt Service System oder Smarten Produkt, die Forderung nach der Individualisierung von Produkten sowie zunehmend komplexe und dynamische Zielsysteme und Rahmenbedingungen im globalen Wettbewerb stellen Entwicklungsteams vor große 
Herausforderungen. Grundsätzliche Weichen werden dabei mit der Anforderungsdefinition in den frühen Phasen der Produktentwicklung gestellt. Anforderungen stellen als spezifische Zielgrößen des Entwicklungsvorhabens gleichzeitig Steuerungsinstrument und Messgröße für alle involvierten Mitarbeiter und das Entwicklungsergebnis dar. Methodisches Vorgehen beim Arbeiten mit Anforderungen ist deshalb ein Schlüsselfaktor für den Erfolg von Entwicklungsvorhaben. Herausforderungen, die dabei bewältigt werden müssen, werden von Methoden des Anforderungsmanagements, der klassischen Konstruktionsmethodik und des Systems Engineering nur in Einzelaspekten adressiert. Eine integrierte Vorgehensweise zur methodischen Unterstützung existiert derzeit nicht.

\section{Zielsysteme für Entwicklungsvorhaben}

Entwicklungsvorhaben werden mit einer Aufgabenstellung - einem internen oder externen Entwicklungsauftrag - gestartet [1]. Der Entwicklungsauftrag muss dafür alle entwicklungsrelevanten Informationen enthalten. In der Praxis reichen diese jedoch je nach Entwicklungskontext (z.B. Unternehmensgröße, Projektgröße, Neuheitsgrad oder das Verhältnis zwischen Auftraggeber und Auftragnehmer) von der mündlichen Auftragserteilung bis hin zu mehreren hundert Seiten umfassenden Aufgabenbeschreibungen und Vertragspapieren [2]. Technische Produktspezifikationen sind essenzielle Bestandteile, um das Zielsystem der Entwicklungsaufgabe festzulegen. Sie allein reichen aber nicht aus, um alle Anforderungen an die Lösung eines Entwicklungsproblems zu erfassen und bestmöglich umzusetzen. Über die darin formulierten produktbezogenen Ziele hinaus ist jedes Entwicklungsvorhaben an Kosten- und Terminziele (Bild 1) sowie limitierende Rahmenbedingungen gebunden. Diese haben einen ebenso großen Einfluss auf die Gestaltungsmöglichkeiten des Produktes.

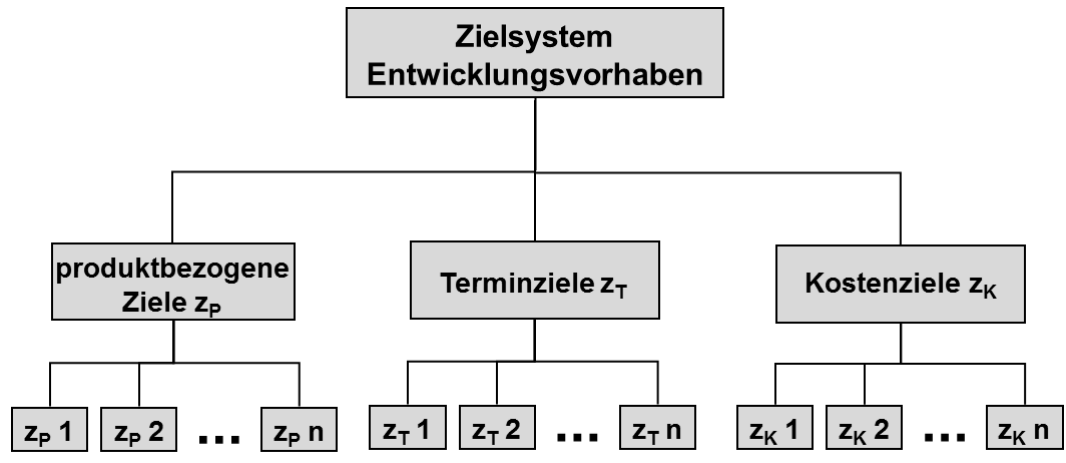

Bild 1: Termin-, Kosten- und produktbezogene Ziele im Zielsystem 
Bei der Umsetzung einer konkreten Lösung treten die Ziele häufig in eine konkurrierende Wechselwirkung [3]. Etwa ein Produkt, das aus Sicherheitsgründen hohen Qualitätsansprüchen genügen muss, kann höhere Materialkosten oder aufwändigere Fertigungs- und Montageprozesse bedingen. Die gleichzeitige Umsetzung zweier oder mehrerer Ziele kann sich gegenseitig ganz ausschließen, beispielsweise wenn verfügbare nachhaltige Materialien für eine ökologischere Ausrichtung nicht die geforderten mechanischen Eigenschaften aufweisen. Die Ziele eines Entwicklungsvorhabens können also nicht unabhängig voneinander für eine gewählte Lösung optimiert werden. Ist die Auflösung entstehender Konflikte auch mittels innovativer Lösungskonzepte nicht möglich, müssen unter Berücksichtigung des spezifischen Entwicklungskontextes Ziele priorisiert und Kompromisse gefunden werden [4]. Diese Kompromissfindung erfordert die Beteiligung aller relevanten Stakeholder und muss bereits in der frühen Planungsphase aktiv angegangen werden, um den hohen Aufwand für die Lösung spät erkannter Zielkonflikte zu vermeiden.

Die Aufgabe des Entwicklungsteams besteht deshalb darin, die Ziele - mitunter (noch) unscharfe Soll-Vorstellungen der Stakeholder [5] - in eindeutige, technisch korrekte und widerspruchsfreie Anforderungen zu übersetzen. Diese stellen über alle Entwicklungsaktivitäten die konkretisierte Arbeitsgrundlage und Vergleichsgröße dafür dar, in wie weit die Entwicklungsziele erreicht werden. Nicht alle Anforderungen und Randbedingungen sowie deren Wechselwirkungen können aber zu Beginn der Entwicklung vollständig erfasst werden. Neben Definitionslücken, d. h. noch nicht (explizit) festgelegten Anforderungen, bestehen in dieser frühen Phase auch Wissenslücken, die erst mit den Erkenntnissen aus der Konkretisierung der Lösung (bekannt als "Koevolution" von Problem und Lösung [6]) geschlossen werden können [7]. Im Verlaufe der Entwicklung werden weitere Rahmenbedingungen, Anforderungen an die technische Umsetzung oder Wechselwirkungen mit anderen Teilsystemen abgeleitet.

\section{Fallstudie: Entwicklung eines mobilen Handrehabilitations-Gerätes}

Anhand eines Anwendungsbeispiels wird veranschaulicht, welche Herausforderungen aufgrund der Unsicherheiten sowie Wechselwirkungen zwischen Anforderungen einerseits und der Dynamik des Zielsystems andererseits für die Anforderungsdefinition entstehen.

Das geförderte Projekt wurde in Kooperation mit einem Unternehmen erstellt, das Rehabilitationsgeräte für die Motorik der Hand entwickelt und vertreibt. Bei Schlaganfall-Patienten treten irreversible Schädigungen im Gehirn auf, die zu motorischen und sensorischen Störungen der Hand führen können. 
Durch intensives Training kann die Bewegungsfähigkeit zumindest teilweise wiederhergestellt werden. Ein Ansatz zur Steigerung der Trainingsintensität ist die roboter- und gerätegestützte Therapie. Das Ziel der Kooperation war die Weiterentwicklung einer ersten Gerätegeneration zur passiven Bewegung einzelner Finger auf vorgegebenen Bewegungsbahnen durch ein innovatives Lösungskonzept. Tabelle 1 zeigt einen Ausschnitt aus den produktbezogenen Zielen, Terminzielen und Kostenzielen dieses Projektes.

Tabelle 1: Ausschnitt aus dem Zielsystem des Anwendungsbeispiels

\begin{tabular}{|l|c|c|}
\hline Ziele & Muss & Soll \\
\hline Produktbezogene Ziele & & \\
\hline Anpassungsmöglichkeit für verschiedene Handgrößen & $\mathrm{x}$ & \\
\hline Ermöglichung definierter Fingerbewegungen und Handauflage & $\mathrm{x}$ & \\
\hline Mobile, autonome Heimanwendung & $\mathrm{x}$ & \\
\hline Verbesserung Design und Ergonomie & $\mathrm{x}$ & \\
\hline Listung im Heil- und Hilfsmittel-Katalog (Krankenkassen) & $\mathrm{x}$ & \\
\hline Anbindung an Serious-Game via Tablet & & $\mathrm{x}$ \\
\hline Terminziele & & \\
\hline Projektlaufzeit 2 Jahre (Förderdauer) & $\mathrm{x}$ & \\
\hline Messetermine (Meilensteine für Prototypen) & $\mathrm{x}$ & \\
\hline Semesterablauf (Integration von Studierendenprojekten) & & $\mathrm{x}$ \\
\hline $\begin{array}{l}\text { Parallelisierung von Design, Steuerungskonzept und Entwick- } \\
\text { lung mechanischer Baugruppen }\end{array}$ & $\mathrm{x}$ & \\
\hline Kostenziel & & \\
\hline Verkaufspreis deutlich geringer als bisheriges Produkt & $\mathrm{x}$ & \\
\hline
\end{tabular}

Die initialen Anforderungen an das zu entwickelnde Gerät wurden durch das Unternehmen definiert bzw. erhoben. Zur Einhaltung des vorgegebenen Terminplans war der agile Entwicklungsprozess gekennzeichnet durch eine Parallelisierung der unterschiedlichen Aktivitäten der Projektbeteiligten, etwa die frühzeitige Durchführung von Design- und Ergonomiestudien und die Herstellung von Prototypen. Bild 2 zeigt schematisch die tatsächlich durchgeführten Entwicklungsaktivitäten, zugeordnet zu den Phasen der VDI 2221. 


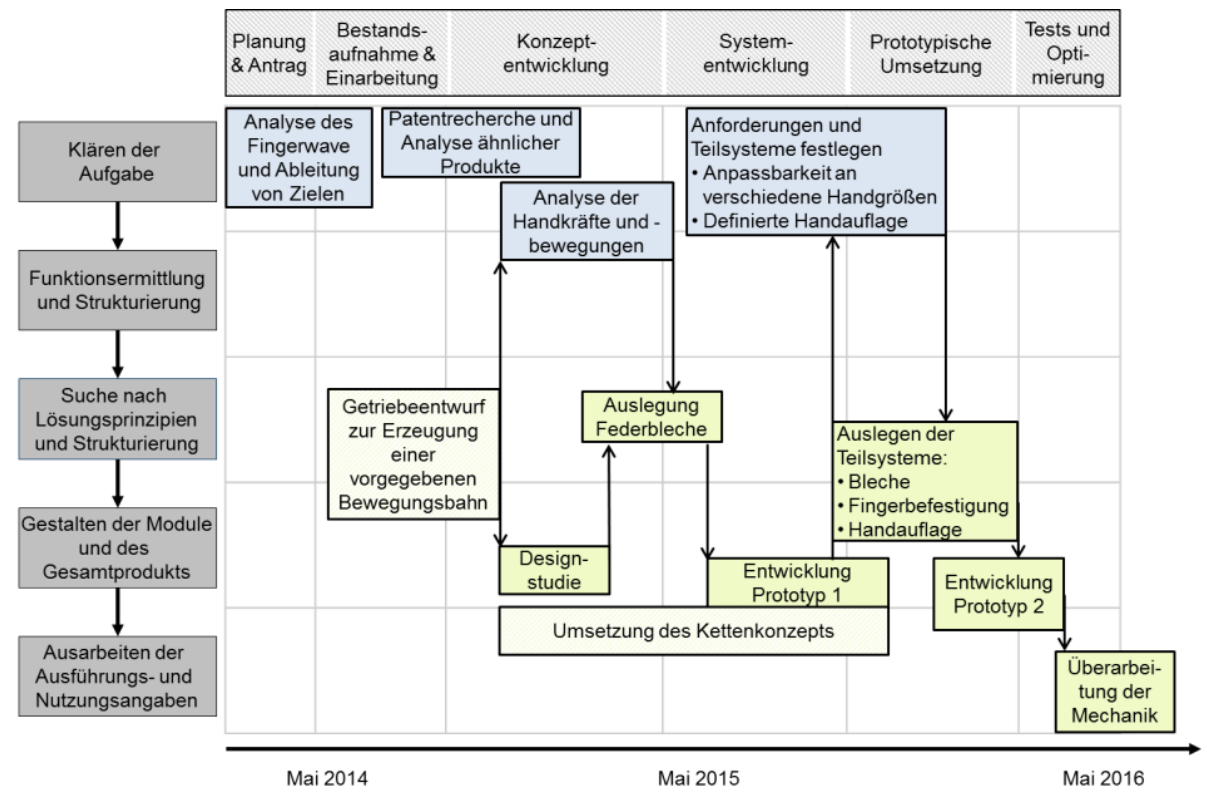

Bild 2: Entwicklungsprozess des Fallbeispiels

In Bezug auf das Arbeiten mit Anforderungen führte die parallelisierte Arbeitsweise dazu, dass wichtige Anforderungen an das Gerät erst spät erkannt werden konnten. Beispielsweise wurde die Kraft, die zur Bewegung der Finger aufgebracht werden muss, zunächst basierend auf einer Messung angenommen, wie viel Kraft einzelne Finger beim Greifen aufbringen können. Auf dieser Grundlage wurde ein Lösungskonzept entwickelt, welches die Bewegung der Finger auf einzelnen Bahnen mittels Energieketten vorsah (Bild 3, Konzept A). Jede einzelne Energiekette wurde durch einen eigenen Rotationsmotor angetrieben, um die Finger unabhängig voneinander unterschiedlich bewegen zu können. Parallel zu der prototypischen Umsetzung dieses Lösungskonzeptes wurden umfassende Kinematik- und Kinetikstudien zur Beweglichkeit der Finger von Patienten durchgeführt. Das Ergebnis zeigte, dass die Handspastik von Patienten mit einer so hohen Fingersteifigkeit einhergeht, dass eine sehr viel gröBere Kraft als bei gesunden Menschen zur Bewegung benötigt wird. Die festgelegte Motorleistung des Prototyps reichte nicht aus, was die Tests mit Probanden bestätigten. Verfügbare Motoren besaßen jedoch entweder nicht die benötigte höhere Leistung, passten nicht in den vorhandenen Bauraum oder lagen nicht in den Zielkosten. Eine Überarbeitung und Fertigstellung dieses Konzeptes ginge mit viel Aufwand einher und musste auch aufgrund der zu hohen resultierenden Produktkomplexität verworfen werden. 


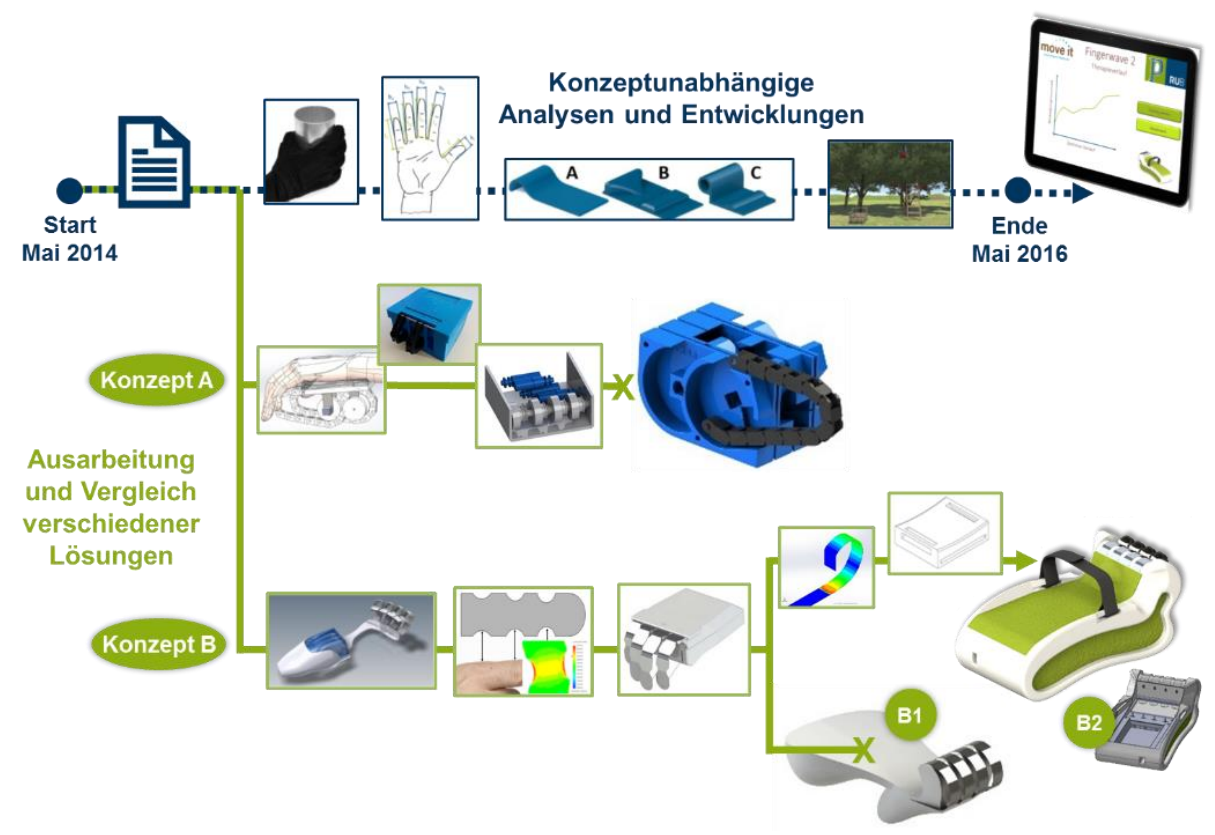

Bild 3: Projektverlauf mit Lösungskonzepten A und B

Parallel dazu wurde ein zweites Lösungskonzept auf Basis einer reinen Design- und Ergonomiestudie entwickelt. Dieses sah die Bewegung der Finger auf Federblechen mit Soll-Biegestellen vor, die anhand anthropometrischer Daten an den Fingergelenken positioniert werden sollten (Bild 3, Konzept B). Diese Studie ergab jedoch eine zu große Varianz in den Gelenkabständen unterschiedlich großer Hände. Außerdem wurde erst spät bedacht, dass die Gelenkabstände an den Fingerauflageflächen (Innenseite) während der Fingerbeugung und -streckung variieren. Aufgrund dessen wurde ein Konzept mit kontinuierlich vorgebogenen Federblechen entwickelt. Diese besitzen außerdem den Vorteil, dass ihre Rückstellkräfte zum passiven Öffnen der Hand (also zur Fingerstreckung) genutzt werden kann. Auf diesen Federblechen gleitende Fingerfixierungen ermöglichen den notwendigen Ausgleich der variierenden Fingerlängen während der Bewegung. Für die Vorbiegung der Federbleche wurden für einen ersten Prototypen Fäden mit Flex-Sensoren eingesetzt. Hier stellten sich insbesondere die Seilzüge als problematisch heraus. Die aufwendige Fertigung der filigranen Mechanik sowie aufgetretene und potenzielle Fehler des Prototyps führten dazu, dass die Seilzüge letztlich durch ein lineares Antriebskonzept ersetzt wurde. Die späte Änderung des Antriebskonzeptes hatte zur Folge, dass in einer weiteren Iteration der Bauraum des Gerätes verlängert werden musste. 
Die angeführten Beispiele verdeutlichen:

- Die technische Lösung wird nicht nur durch produktbezogene Ziele, sondern auch durch Termin- und Kostenziele sowie Rahmenbedingungen determiniert. Durch das Ermitteln der initialen Anforderungsbasis wird das Fundament für das methodische Konstruieren gelegt.

- Nicht alle Anforderungen können direkt zu Beginn (umfassend, vollständig, korrekt) definiert werden. Die Parallelisierung von Entwicklungsaktivitäten sowie die Koevolution von Problem und Lösung können über den gesamten Produktentwicklungsprozess zur Änderung bereits festgelegter sowie zum Erkennen neuer Anforderungen führen.

- Konflikte zwischen Zielen bzw. Anforderungen entstehen erst mit der Wahl einer konkreten Lösung. Sind sie durch konzeptuelle/konstruktive Änderungen nicht zu lösen, müssen durch Priorisierung der Anforderungen Kompromisse gefunden werden.

Daher werden im Folgenden methodische Ansätze im Hinblick auf ihre Eignung zum Umgang mit diesen charakteristischen Herausforderungen beim Arbeiten mit Anforderungen in der Produktentwicklung diskutiert.

\section{Methodische Ansätze für das Arbeiten mit Anforderungen}

In der Konstruktionsmethodik wird der Definition von Anforderungen aufgrund ihres maßgeblichen Einflusses auf die gesamte Produktentwicklung eine zentrale Rolle zugeschrieben. Allen einflussreichen Vorgehensmodellen ist gemein, dass der erste Schritt aus der Anforderungsdefinition besteht. Jedoch fehlen in den Vorgehensmodellen detaillierte Beschreibungen dieser Aufgabe [8] und es entsteht der Eindruck, dass die Anforderungsdefinition weitestgehend abgeschlossen ist bzw. sein muss, bevor mit der systematischen Suche nach Lösungen begonnen werden kann [9]. Dadurch kann das Arbeiten mit Anforderungen anstelle der Zuordnung zu den Querschnittsfunktionen als einmalige Aktivität beim Entwicklungsstart fehlinterpretiert werden.

In der Regel wird in der Produktentwicklungsliteratur auf Aktivitäten und Methoden des Anforderungsmanagements verwiesen. Diese können - wie häufig aus dem Englischen übernommen - in "Requirements Engineering", als dem Prozess der Anforderungserfassung und -strukturierung [10], und "Requirements Management", als dem Prozess der Datenerfassung und fortlaufenden Pflege der Anforderungen im Projekt [11], aufgeteilt werden. Unabhängig vom Begriffsverständnis, das aufgrund unterschiedlicher Interpretationen und 
Übersetzungen uneinheitlich gehandhabt wird, können folgende grundlegenden Aktivitäten des Arbeitens mit Anforderungen unterschieden werden: Anforderungen ermitteln, spezifizieren, strukturieren, analysieren, dokumentieren, ändern, versionieren, rückverfolgen sowie prüfen und kommunizieren [12].

Zur Entwicklung einer initialen Anforderungsbasis sollten im ersten Ansatz immer vorhandene Quellen und Dokumente in Hinblick auf die Vorgabe von Anforderungen geprüft werden. Dies können neben dem Entwicklungsauftrag oder dem Lastenheft auch Vertragsdokumente wie Leistungs- und Lieferpläne oder Gesprächsprotokolle umfassen. Häufig gibt es im Unternehmen bereits ein vergleichbares Vorgängerprodukt (vgl. Produktgenerationenentwicklung nach [13]), dessen Anforderungen für das neue Entwicklungsvorhaben geprüft und angepasst werden können. Die sich daraus ergebenden ersten Anforderungen müssen mit Hilfe systematischer Ansätze iterativ vervollständigt werden. Dabei helfen unterschiedliche Methoden und Checklisten, die neben dem Kunden weitere Stakeholder außerhalb und innerhalb des Unternehmens aus unterschiedlichen Produktlebensphasen berücksichtigen. Eine bekannte Checkliste ist die "Hauptmerkmalliste" nach [14], die physikalische und geometrische Eigenschaften umfasst, und von [1] zu einer erweiterten Kriterienliste um Beurteilungskriterien aus anderen Lebenszyklusphasen sowie organisatorische und marktrelevante Aspekte ergänzt wurde. Weit verbreitet für die Anforderungsermittlung sind außerdem erfahrungs- oder meinungsbasierte Methoden (wie z.B. Fragebögen, Benchmarking, Prototypenbeobachtung) und Kreativitätsmethoden (z.B. 6-3-5 Methode, Szenario-Technik). Bei der Ermittlung der Kundenanforderungen ist es von zentraler Bedeutung, auch implizite Anforderungen zu finden, die z.B. selbstverständlich erwartet werden oder nicht bewusst sind und deshalb nicht explizit genannt werden. Implizite Anforderungen, die oft erst nach dem Markteintritt des Produkts identifiziert werden, lassen sich methodisch ermitteln. Bekannt sind z. B. Benchmarking, Beschwerdesysteme, Gebrauchstests, Interviews mit Lead Usern oder Focus Groups, Lost CustomerUmfragen und Win/Loss Reports [15]. Vage Forderungen und Wünsche - wie häufig zunächst durch Kunden formuliert - müssen für die Entwicklung als präzise, technische Anforderungen spezifiziert werden. Umgekehrt können Stakeholder bereits konkrete Anforderungen mit sehr spezifischen Zielwerten angeben - typisch sind hier Fertigungsvorgaben durch Systemlieferanten - und damit die umsetzbaren Lösungsmöglichkeiten einschränken. Ist dagegen ein Spielraum bei der Erreichung von Zielwerten zulässig, muss die Anforderung zur weiteren Bearbeitung entsprechend spezifiziert werden, etwa durch Angabe eines Wertebereiches oder Grenzwertes statt eines festen Punktwertes [12]. Allgemein müssen bei der Spezifizierung von Anforderungen Qualitätskriterien eingehalten werden, um eine zielgerichtete Entwicklung zu ermöglichen und aufwendige Iterationen zu reduzieren. Nach [11] sollten Anforderungen 
eindeutig, gültig/vereinbart/aktuell, korrekt, realisierbar, rückverfolgbar, verifizierbar, verständlich und vollständig sein. Unterstützung für die Anforderungsspezifikation bieten Glossare, Satzschablonen oder syntaktische Satzbaumuster wie das des VDA [16]. Die Strukturierung der ermittelten und spezifizierten Anforderungen ist notwendig, um Doppelungen, fehlende Informationen, aber auch Widersprüche zwischen Zielen oder Anforderungen zu finden. Dafür kann es nützlich sein, die Anforderungen hinsichtlich folgender Kriterien (Zusatzinformationen) zu kategorisieren: Funktionalität (funktional vs. nichtfunktional), Gegenstand (produktspezifisch vs. produktneutral), Verbindlichkeit (Forderung vs. Wunsch), Messbarkeit (qualitativ vs. quantitativ), Bewusstheit (explizit vs. implizit), Kritikalität (aktiv vs. passiv wechselwirkende), Einfluss auf Kundenzufriedenheit (Basis-, Leistungs-, Begeisterungseigenschaft), Stakeholder (intern vs. extern), Toleranzbereich (Punkt-, Grenz- und Bereichsanforderung) und Lösungseingrenzung (lösungsneutral vs. lösungsspezifisch) der Anforderung [in Anlehnung an 12]. Eine weitere wichtige Information für die Strukturierung ist die Festlegung der Verantwortlichkeiten.

Ziele bei der Analyse von Anforderungen sind die Vervollständigung der Anforderungsbasis sowie das Identifizieren und Auflösen von Zielkonflikten durch Priorisierung der Anforderungen. Dafür können die zur Strukturierung aufgezählten Kriterien genutzt werden. Etablierte Methoden für die Spezifizierung, Strukturierung und Analyse der Anforderungen sind z. B. das House of Quality, das KANO-Modell oder die Conjoint-Analyse. Da mit zunehmendem Entwicklungsfortschritt neue Erkenntnisse gewonnen werden, muss zu jedem Zeitpunkt im Projekt der gültige Stand der Anforderungen dokumentiert sowie Änderungen über die gesamte Projektlaufzeit gekennzeichnet und nachverfolgt werden. Unterstützung dabei bieten etablierte Tools wie DOORS.

Methoden des Anforderungsmanagements bieten eine fokussierte Sicht auf Anforderungen. Ihre dynamischen Wechselwirkungen (vgl. Bild 4) mit dem Entwicklungsprozess und dem Entwicklungsergebnis (von Zwischenergebnissen bis zum fertigen Produkt) werden im Systems Engineering dargestellt. Zurückgehend auf die zuerst von Ropohl vorgenommenen Definitionen von Handlungssystem, Sachsystem und Zielsystemen entwickelte Negele ein Beschreibungsmodell für die Produktentwicklung bestehend aus Ziel-, Objekt-, Prozess und Handlungssystem [17]. Dieses wurde weiterentwickelt zum integrierten Produktentstehungsmodell (iPeM) [18], in dem Prozess- und Handlungssystem zusammengefasst sind. Gemeinsam ist den Definitionen, dass das Zielsystem alle expliziten Ziele und Anforderungen einschließlich deren Abhängigkeiten und Randbedingungen beinhaltet, die durch das Handlungssystem in ein konkretes Sachsystem überführt werden. 


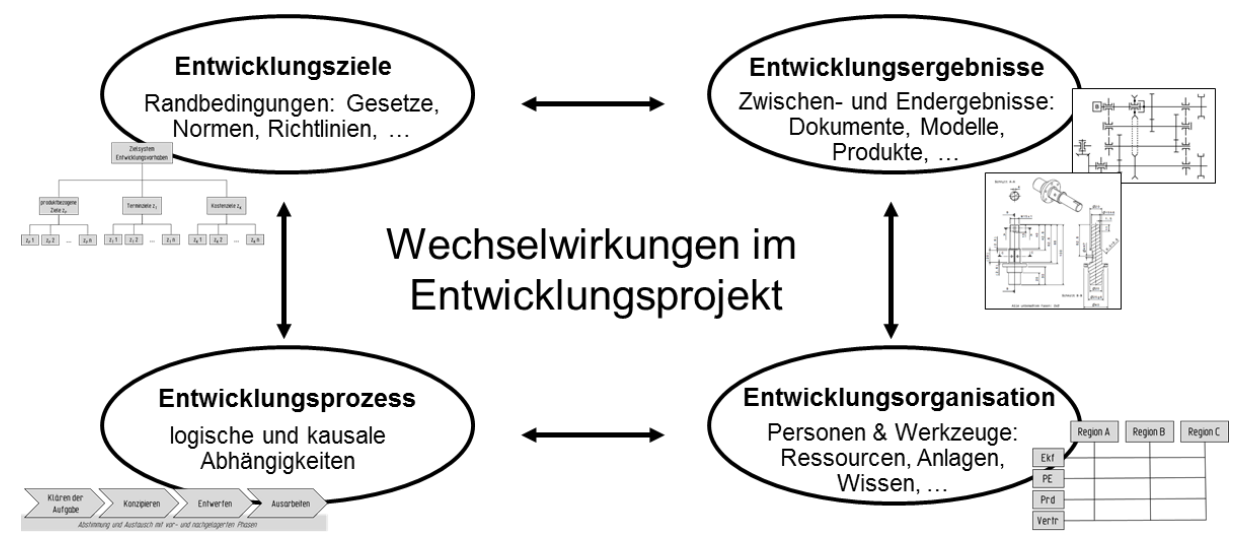

Bild 4: Wechselwirkender Einfluss von Entwicklungszielen, -ergebnissen, -prozess und -organisation

Die Relevanz dieser Wechselwirkungen zeigt Neumann auf, indem er daraus eine modellbasierte Vorgehensweise zur risikoorientierten Entwicklung innovativer Produkte herleitet [19]. Er zeigt auf, dass sich Unsicherheiten eines Partialsystems in die anderen fortpflanzen. Ebenso wirken sich alle möglichen Maßnahmen des Risikomanagements auf alle drei Partialsysteme aus. So könnte eine Auslegungsvorgabe im Zielsystem (wie etwa die benötigte Kraft in der Fallstudie) zu aufwendigen Tests (Handlungssystem) mit Prototypen (Sachsystem) führen, um diese initiale Annahme im Zielsystem zu bestätigen bzw. korrigieren. Es könnte aber auch das Risiko eingegangen werden, ohne überprüfende Aktivitäten und Testobjekte an der Auslegungsvorgabe festzuhalten.

\section{$5 \quad$ Diskussion und Fazit}

Die Entwicklung von Anforderungen ist keine triviale Aufgabe, weil gerade zu dem frühen Zeitpunkt, an dem diese Aktivität ansetzt, Wissen und Informationen über das Entwicklungsvorhaben noch mit hohen Unsicherheiten behaftet sind. Typische auftretende Probleme werden anhand einer Fallstudie beschrieben. Das Fallbeispiel verdeutlicht, dass viele wichtige Definitions- und Wissenslücken erst mit den Erkenntnissen aus der Konkretisierung der Lösung („Koevolution" von Problem und Lösung) geschlossen werden können. Für ein methodisches Vorgehen dürfen die zu definierenden Anforderungen deshalb nicht isoliert betrachtet werden. Stattdessen müssen auch ihre Wechselwirkungen mit den projektspezifischen Potenzialen des Entwicklungsprozesses und des Entwicklungsergebnisses Berücksichtigung finden. Eine systemtheoretische Betrachtung der Produktentwicklung, mit der diese Wechselwirkungen erfasst und abgebildet werden können, ist deshalb eine notwendige Ergänzung zu den 
Methoden des Anforderungsmanagements. Diese integrierte Sichtweise wurde in Neumanns Vorschlag zur Identifizierung und Handhabung von Risiken in der Produktentwicklung für diskrete Entwicklungssituationen umgesetzt. Aufgrund der enormen zu verarbeitenden Datenmenge wäre eine dynamische Anwendung zu komplex und bietet zunächst nur in kritischen Entscheidungssituationen Unterstützung. Die Entwicklung einer Vorgehensweise zum Umgang mit den charakteristischen Zielkonflikten beim Entwickeln ist somit ein zentrales Ziel für das Arbeiten mit Anforderungen in der Produktentwicklung.

\section{Literatur}

[1] Nagarajah, A.: Methodisches Klären und Präzisieren der Aufgabenstellung. In: Feldhusen, J. und Grote, K.-H. (Hrsg.): Pahl/Beitz Konstruktionslehre: Methoden und Anwendung erfolgreicher Produktentwicklung. Springer-Verlag, 2013.

[2] Jakoby, W.: Projektmanagement für Ingenieure. Ein praxisnahes Lehrbuch für den systematischen Projekterfolg. 3. Aufl., Springer-Vieweg Verlag, Wiesbaden, 2015.

[3] Salado, A. und Nilchiani, R.: "The concept of order of conflict in requirements engineering", IEEE Systems Journal, Vol. 10., No. 1, März 2014, S. 25-35.

[4] Song, Y.-W., Windheim, M. und Bender, B.: "Challenges in the definition and prioritisation of requirements: A case study". In DS92: Proceedings of the DESIGN 2018 15th International Design Conference, 2018

[5] Ehrlenspiel, K. und Meerkamm, H.: Integrierte Produktentwicklung: Denkabläufe, Methodeneinsatz, Zusammenarbeit. Carl Hanser Verlag GmbH Co KG., 2017.

[6] Dorst, K. und Cross, N.: "Creativity in the design process: co-evolution of problem-solution". Design studies, 22(5), 2001, S. 425-437.

[7] Hastings, D. und McManus, H.: „A Framework for Understanding Uncertainty and its Mitigation and Exploitation in Complex Systems". In: Engineering Management Review, Vol. 34, No. 3, 2006.

[8] Chakrabarti, A., Morgenstern, S., and Knaab, H.: "Identification and application of requirements and their impact on the design process: a protocol study". Research in engineering design, 15(1), 2004, S. 22-39. 
[9] Hansen, C. T. and Andreasen, M. M.: "Specifications in early conceptual design work". In: 16th International Conference on Engineering Design (pp. 1-12). Ecole Central Paris \& The Design Society, 2007.

[10] Marcus Grande: 100 Minuten für Anforderungsmanagement. Kompaktes Wissen nicht nur für Projektleiter und Entwickler, 2. Auflage, Springer Vieweg, 2011.

[11] Pohl, K. und Rupp, C.: Basiswissen Requirements Engineering. Aus- und Weiterbildung zum "Certified Professional for Requirements Engineering", Heidelberg, dpunkt.verlag GmbH, 2015.

[12] Baumgart, I.: Requirements Engineering. In: Lindemann, U. (Hrsg.): Handbuch Produktentwicklung. Carl Hanser Verlag GmbH Co KG, 2016.

[13] Albers, A.; Bursac, N. and Wintergerst, E.: "Product generation development - importance and challenges from a design research perspective", In: New developments in mechanics and mechanical engineering, 2015.

[14] Pahl, G. und Beitz, W.: Konstruktionslehre. Grundlagen erfolgreicher Produktentwicklung - Methoden und Anwendung. Springer Verlag, 2007.

[15] Ahrens, G.: Das Erfassen und Handhaben von Produktanforderungen. TU Berlin, Dissertation, 2000.

[16] VDA 2006: Geometrische Produktspezifikation. Oberflächenbeschaffenheit - Regeln und Verfahren zur Beurteilung der Oberflächenbeschaffenheit, VDA-Verlag, 2003.

[17] Negele, H.: Systemtechnische Methodik zur ganzheitlichen Modellierung am Beispiel der integrierten Produktentwicklung, TU München, Dissertation, 1998.

[18] Albers, A., Reiß, N., Bursac, N., Richter, T.O.: „iPeM - Integrated Product Engineering Model in Context of Product Generation Engineering". In: Procedia CIRP 50, 2016.

[19] Neumann, M.: Ein modellbasierter Ansatz zur risikoorientierten Entwicklung innovativer Produkte. Ruhr-Universität Bochum, Dissertation, 2017. 